\title{
Long-term outcome and prognostic nomogram for intrahepatic cholangiocarcinoma after thermal ablation: a retrospective study
}

\author{
Yang Wang ${ }^{1,2 \#}$, Zhuhui Yuan ${ }^{1 \#}$, Bozhi Liu ${ }^{1 \#}$, Hui Chen ${ }^{3}$, Tian Yin ${ }^{2}$, Jiasheng Zheng ${ }^{2}$, Wei Li ${ }^{1}$ \\ ${ }^{1}$ Cancer Center, Beijing Ditan Hospital, Capital Medical University, Beijing, China; ${ }^{2}$ Center of Interventional Oncology and Liver Diseases, Beijing \\ You'an Hospital, Capital Medical University, Beijing, China; ${ }^{3}$ School of Biomedical Engineering, Capital Medical University, Beijing, China \\ Contributions: (I) Conception and design: W Li, J Zheng, Y Wang; (II) Administrative support: W Li, J Zheng; (III) Provision of study materials or \\ patients: Y Wang, Z Yuan, B Liu, T Yin; (IV) Collection and assembly of data: Y Wang, Z Yuan, B Liu; (V) Data analysis and interpretation: Y Wang, \\ Z Yuan, B Liu; (VI) Manuscript writing: All authors; (VII) Final approval of manuscript: All authors. \\ \#These authors contributed equally to this work. \\ Correspondence to: Dr. Wei Li. Cancer Center, Beijing Ditan Hospital, Capital Medical University, 8 Jingshundong Street, Chaoyang District, Beijing, \\ China. Email: vision988@126.com; Dr. Jiasheng Zheng. Center of Interventional Oncology and Liver Diseases, Beijing You'an Hospital, Capital \\ Medical University, 8 Xitoutiao, Youwai Street., Fengtai District, Beijing, China. Email: zhengjiasheng6@sina.com.
}

\begin{abstract}
Background: To evaluate the efficacy of thermal ablation and transcatheter arterial chemoembolization (TACE) in treating intrahepatic cholangiocarcinoma (ICC), and to propose a prognostic nomogram to predict overall survival (OS) after ablation.

Methods: ICC patients ( $\mathrm{n}=58$ ) undergoing percutaneous CT-guided ablation with or without preablation TACE at our institute from 2009 to 2013 were enrolled in the primary cohort. All treatments were performed under conscious sedation and local anesthesia. Prognostic factors for OS were identified to establish the nomogram. The nomogram was accessed by the concordance index (C-index) and calibration curve, and compared with 11 currently available prognostic systems. The nomogram was further evaluated in a validation cohort $(n=19)$ of patients with ICC who underwent thermal ablation and TACE from 2014 to 2016.

Results: In the primary cohort, the median OS was 17.9 months and the 1-, 3-, 5-year OS rates were $69.6 \%, 29.5 \%, 23.6 \%$, respectively. Three single independent predictors (number of tumors, vascular invasion and lymph node metastasis) for OS were identified by multivariate analysis and used to formulate the nomogram. The C-index of the nomogram was 0.834 [95\% confidence interval (CI), 0.765 to 0.904], significantly higher than that of ten current prognostic systems for ICC, and similar with that of the Wang nomogram. The performance of the proposed nomogram was also confirmed in the validation cohort (C-index, 0.839).
\end{abstract}

Conclusions: Thermal ablation appears to be effective for ICC patients. The proposed nomogram is helpful for predicting patient survival after ablation treatment.

Keywords: Intrahepatic cholangiocarcinoma (ICC); ablation; TACE; overall survival (OS); nomogram

Submitted Jun 09, 2020. Accepted for publication Oct 09, 2020.

doi: $10.21037 /$ tcr-20-2299

View this article at: http://dx.doi.org/10.21037/tcr-20-2299

\section{Introduction}

Intrahepatic cholangiocarcinoma (ICC) is a malignant tumor originating from the epithelial cells of second- and higher-order branches of the intrahepatic bile duct (1).
While cholangiocarcinoma accounts for $10-15 \%$ of all hepatobiliary malignancies, ICC accounts for approximately $8 \%$ of cholangiocarcinomas (2,3). Morbidity and mortality due to ICC have been increasing worldwide, but the reasons remain uncertain (4). Surgical resection is considered 
the first-line treatment for ICC (5). But prognosis after hepatectomy remains unsatisfactory, with 5-year overall survival (OS) rates reported to be 18-43.2\% (6-13). Moreover, more than two-thirds of patients are not suitable for resection at diagnosis due to a lack of symptoms until advanced stage. Historically, prognosis of untreated patients with unresectable ICC is poor, with median OS of merely 3 months (14).

Thermal ablation, including radiofrequency ablation (RFA) and microwave ablation (MWA), has gained wide acceptance in the management of hepatocellular carcinoma (HCC) (15). The heating process of RFA is more moderate and the ablative zone is easier to control; while MWA is more powerful and capable of yielding larger ablative volumes in a shorter time. Transcatheter arterial chemoembolization (TACE) is a safe palliative treatment recommended for intermediate-stage HCC in the Barcelona clinic liver cancer (BCLC) staging system and is often performed prior to thermal ablation, as well as after failure of standard first-line therapies (16). However, the efficacy of thermal ablation and TACE in ICC patients has not been well studied. Currently, thermal ablation is mainly applied in unresectable ICCs or post-surgery relapse, with 1- and 5 -year OS rates to be $\sim 89 \%$ and $24 \%$, respectively $(17,18)$.

ICC differs from HCC and other types of cholangiocarcinoma in terms of clinical manifestations, imaging characteristics and therapeutic management. Several distinct prognostic staging systems have been proposed for patients with ICC treated by surgery, including six staging systems [the $7^{\text {th }}$ edition of the American Joint Committee on Cancer (AJCC) TNM classification (19), Nathan (9), Okabayashi (7), LCSGJ (20), Uenishi (11) and Sakamoto (21) staging systems], two score systems [the Fudan score (10) and the Zhou score (22) system] and three prognostic nomograms [the Wang (6), Hyder (12) and Yeh (13) nomograms]. However, all of these prognostic systems are based on data from patients undergoing resection. None of them have been validated in patients treated by thermal ablation and TACE.

The aim of this study was (I) to evaluate the efficacy of thermal ablation and TACE in patients with ICC and (II) to develop a prognostic nomogram for estimating OS of ICC patients treated with these modalities. The prognostic accuracy of the proposed nomogram was evaluated and compared with currently available prognostic systems.

We present the following article in accordance with the TRIPOD reporting checklist (available at http://dx.doi. org/10.21037/tcr-20-2299).

\section{Methods}

\section{Patient cohort}

A total of 77 patients with resectable $(n=34)$ or unresectable $(n=43)$ ICCs treated by ablation procedures from January 1, 2009 to December 31, 2016 were identified at Beijing You'an Hospital. Inclusion criteria: (I) ICC was confirmed by pathological biopsy; (II) patients with unresectable ICC, or patients with resectable ICC but refused surgery and required for minimally invasive treatment, or patients failed from previous treatments, such as tumor recurrence and progression after resection or chemotherapy. The exclusion criteria were as follows: severe underlying diseases; platelet count less than $50 \times 10^{3} / \mu \mathrm{L}$; untreatable extrahepatic metastasis; patient refusal. Unresectable was defined as follows: distant metastatic disease; extensive local involvement (bilateral and/or contralateral vascular and/or biliary involvement); inadequate future liver remnant (23). Lymph node metastasis was retrospectively diagnosed by sequential CT imagings based on typical appearances such as increasing size and numbers within weeks or months, or representative manifestations on FDG-PET/PET-CT. Vascular invasion was assessed by pre-operative CT imaging, and presented as cordlike or columnar masses in the hepatic vein or portal vein, usually originating from the tumor mass. The tumor thrombus could show similar enhancement pattern as HCC with hyper attenuation at the arterial phase and rapid washout at the venous phase. A total of 34 patients had resectable disease but opted for ablation instead of surgery. Thirtythree of them refused surgery due to advanced age and unwillingness to take risks of anesthesia and surgery; while the other patient had tumor recurrence after previous surgery.

The primary cohort of this study was composed of 58 patients from 2009 to 2013 and was analyzed to establish a prognostic model. The other 19 patients undergoing ablation from 2014 to 2016 formed the validation cohort. The study covered the data analysis of all patients.

The study was conducted in accordance with the Declaration of Helsinki (as revised in 2013) and the Harmonized Tripartite Guideline for Good Clinical Practice from the International Conference on Harmonization. This study was reviewed and approved by the Beijing You'an Hospital Ethics Committee [approval number 2017(28)]. All patients enrolled completed the informed consent form. 


\section{Pre-ablation TACE}

For every patient, a contrast-enhanced CT imaging was performed before treatment and was used as the reference image. The decision to treat patients by thermal ablation (RFA or MWA) with or without pre-ablation TACE were determined by the multidisciplinary tumor board discussions. For tumors with obscure margin on CT scanning or with large tumor size and multiple lesions, pre-ablation TACE was recommended. TACE was performed under local anesthesia. A 5-F pigtail catheter was introduced through the femoral artery, and the survey of arteries was performed. The chemotherapeutic agents [including epirubicin $(10 \mathrm{mg})$ and arsenic trioxide $(10 \mathrm{mg})$ ] were injected into the tumor feeding arteries. Then lipiodol (4-10 mL) and gelatin sponge particles $(25-100 \mathrm{mg}$ ) were sequentially injected. The doses of lipiodol and particles were depending on the size of tumor feeding arteries.

\section{Ablation procedures and follow-up}

Percutaneous ablations were performed within 2 weeks after TACE. Patients were under conscious sedation and local anesthesia. RFA (Covidien, Shanghai, China or RITA Medical Systems, Mountain View, CA, USA) or MWA (Qinghai Ltd., Nanjing, China) were used to generate a favorable necrosis zone at the tumor site. For large tumors and those adjacent to big vessels, MWA was preferred; while RFA was more frequently applied to treat the tumors near important tissues such as gallbladder or colon. Probes targeting at the precise tumor position were navigated by CT imaging (Toshiba, Tokyo, Japan). The path of percutaneous puncture was designed with the help of radiopaque marker on the skin and instant computed tomography scan. A $22-G$ needle was used to lead the antenna/electrode to the target. The angle and position of the antenna/electrode were confirmed and adjusted based on intra-operative CT scans. Until the antenna/electrode was advanced up to the tumor, proper ablative parameter and duration time were designed to achieve maximized necrosis of the tumor. The needle tract was also ablated to prevent hemorrhage and needle tract tumor dissemination. After ablation, an instant contrast-enhanced CT scanning was performed to evaluate the ablation zone and possible complications. For post-ablation residual tumor or tumor recurrence, an additional session of ablation would be arranged.

Follow-up imaging (contrast-enhanced CT or magnetic resonance imaging) was performed at a 4-week interval for the first 3 months and every 3 months thenceforth regularly. Follow-up was ended on April 15, 2020. If recurrence was found, salvage ablation was performed. Image findings were interpreted by two radiologists to evaluate technique effectiveness and relapse. OS was defined as the time interval between ablation accomplishment and patient death. Progression-free survival (PFS) was defined as the time interval between ablation accomplishment and detection of tumor recurrence, progression or metastases.

\section{Statistical analysis}

Differences of demographic and clinical characteristics between the primary cohort and validation cohort were compared using SPSS 17.0 for Windows (SPSS, RRID:SCR_002865). The OS and PFS rates were calculated by Kaplan-Meier method. If median survival time was not reached, then the $75 \%$ survival time was calculated. The $75 \%$ survival time was defined as the amount of time after which $25 \%$ of the patients died (75\% patients survived). Prognostic factors were identified by univariate analysis and multivariate analysis. Cox proportional hazards regression model was used for further analysis of variables which were significantly related with OS $(\mathrm{P}<0.05)$ at univariate analysis. The final model selection was performed by a bidirectional elimination process with the Akaike information criterion (24).

Based on the results of multivariate analysis, a nomogram to predict OS was formulated by using the package of rms in $\mathrm{R}$ version 3.3.1 (http://www.r-project.org/). Concordance index (C-index) was calculated to estimate the performance of the nomogram, and calibration curves for predicting patient survival were plotted. The nomogram was compared with current 11 staging systems by C-index calculated by using the rcorrp.cens package in Hmisc in R. Larger C-index indicated better prognostic predictive accuracy. As for the validation of the nomogram, Cox regression was performed using total points calculated by the proposed nomogram in the validation cohort as an independent factor. The $\mathrm{C}$-index were calculated based on the regression analysis to measure the performance of the nomogram. $\mathrm{P}<0.05$ was considered statistically significant.

\section{Results}

\section{Demographic and clinical characteristics}

A total of 58 and 19 patients with pathologically confirmed 
ICCs were continuously enrolled in the primary and validation cohort, respectively. Two hundred and twenty-six tumors (173 in the primary group and 53 in the validation group) underwent 257 sessions of ablation. Seven patients had extra hepatic metastases, and metastases were treated by thermal ablation or radiotherapy. Five patients suffered post-resection tumor recurrence, and were treated by thermal ablation with or without pre-ablation TACE at our institute. The time interval between ablation and primary therapy was ranging from 6 months to 3 years. Mean followup was 14 months, ranging from 4 to 69 months. A total of 10 patients (13\%) were lost to follow up. Among the seven patients with extrahepatic metastasis, four of them were lost to follow up. There were no significant differences between two cohorts at baseline, except in prothrombin time (PT), which was however within normal limits in both groups. Details of the demographic and clinical characteristics of patients in the primary and validation cohorts are listed in Table 1.

\section{Complications}

Minor complications following TACE included mild hepatalgia $(n=11)$, slight fever $(n=7)$, and hypertension $(n=4)$. No major complications were observed for TACE procedures. Following thermal ablation, one patient had a major complication of biliary tract infection, which required antibiotics treatment and prolonged hospital stay. Minor complications after thermal ablation included transient hepatic dysfunction $(n=12)$, hepatalgia $(n=8)$, nausea $(n=3)$ and fever $(n=2)$.

\section{OS and PFS of the primary cohort}

A total of 37 cancer-related deaths were recorded. In the primary cohort of 58 ICC patients, the median OS was 17.9 months and the 1-, 3-, and 5-year OS rates were $69.6 \%, 29.5 \%$, and $23.6 \%$, respectively. For patients with resectable ICC, median OS was 27.8 months, and the 1-, 3 -, and 5 -year OS rates were $86.5 \%, 48.1 \%$, and $38.5 \%$, respectively. For those with unresectable ICC, median OS was 12.7 months, and the 1-year OS rate was 50.6\%, significantly lower than that of patients with resectable ICC $(\mathrm{P}<0.001)$. Median PFS in the primary cohort was 3.4 months, and the 1-, 3-, and 5-year PFS rates were $14 \%$, $6.2 \%$ and $0 \%$, respectively.
Table 1 Demographics and characteristics of primary cohort and validation cohort with ICC

\begin{tabular}{|c|c|c|c|}
\hline $\begin{array}{l}\text { Demographics and } \\
\text { characteristics }\end{array}$ & $\begin{array}{c}\text { Primary } \\
\text { cohort }(n=58)\end{array}$ & $\begin{array}{c}\text { Validation } \\
\text { cohort }(n=19)\end{array}$ & $\begin{array}{l}\mathrm{P} \text { value of } \\
\text { difference }\end{array}$ \\
\hline \multicolumn{4}{|c|}{ Categorical variables, n (\%) } \\
\hline Gender & & & 0.387 \\
\hline Male & $41(70.7)$ & $16(84.2)$ & \\
\hline Female & $17(29.3)$ & $3(15.8)$ & \\
\hline Liver cirrhosis & & & 0.793 \\
\hline No & $34(58.6)$ & $12(63.2)$ & \\
\hline Yes & $24(41.4)$ & $7(36.8)$ & \\
\hline HBsAg (serum) & & & 0.428 \\
\hline Negative & $29(50.0)$ & $7(36.8)$ & \\
\hline Positive & $29(50.0)$ & $12(63.2)$ & \\
\hline Anti-HCV & & & 1 \\
\hline Negative & $52(89.7)$ & $17(89.5)$ & \\
\hline Positive & $6(10.3)$ & $2(10.5)$ & \\
\hline Pre-operative TACE & & & 0.980 \\
\hline No & 7 (12.1) & $3(15.8)$ & \\
\hline Yes & $51(87.9)$ & $16(84.2)$ & \\
\hline Prior liver resection & & & 0.174 \\
\hline No & $56(96.6)$ & $16(84.2)$ & \\
\hline Yes & $2(3.4)$ & $3(15.8)$ & \\
\hline Prior chemotherapy & & & 0.681 \\
\hline No & $52(89.7)$ & $16(84.2)$ & \\
\hline Yes & $6(10.3)$ & $3(15.8)$ & \\
\hline Prior radiotherapy & & & 0.438 \\
\hline No & $50(86.2)$ & $18(94.7)$ & \\
\hline Yes & $8(13.8)$ & $1(5.3)$ & \\
\hline Ablation technique & & & 0.579 \\
\hline RFA & $23(39.6)$ & 7 (36.9) & \\
\hline MWA & $24(41.4)$ & $10(52.6)$ & \\
\hline Both & $11(19.0)$ & $2(10.5)$ & \\
\hline Vascular invasion & & & 0.85 \\
\hline No & $35(60.3)$ & $11(57.9)$ & \\
\hline Yes & $23(39.7)$ & $8(42.1)$ & \\
\hline
\end{tabular}

Table 1 (continued) 
Table 1 (continued)

\begin{tabular}{|c|c|c|c|}
\hline $\begin{array}{l}\text { Demographics and } \\
\text { characteristics }\end{array}$ & $\begin{array}{c}\text { Primary } \\
\text { cohort }(n=58)\end{array}$ & $\begin{array}{c}\text { Validation } \\
\text { cohort }(n=19)\end{array}$ & $\begin{array}{l}\mathrm{P} \text { value of } \\
\text { difference }\end{array}$ \\
\hline Tumor location & & & 0.755 \\
\hline Right lobe & $33(56.9)$ & $10(52.6)$ & \\
\hline Left lobe & $9(15.5)$ & $2(10.5)$ & \\
\hline Bilobed & $16(27.6)$ & 7 (36.9) & \\
\hline \multicolumn{2}{|c|}{ Extrahepatic metastasis } & & 0.834 \\
\hline No & $52(89.7)$ & $18(94.7)$ & \\
\hline Yes & $6(10.3)$ & $1(5.3)$ & \\
\hline \multicolumn{2}{|c|}{ Lymph node metastasis } & & 1.000 \\
\hline No & $47(81.0)$ & $15(78.9)$ & \\
\hline Yes & $11(19.0)$ & $4(21.1)$ & \\
\hline Resectable & & & 0.459 \\
\hline Yes & $27(46.6)$ & $7(36.8)$ & \\
\hline No & $31(53.4)$ & $12(63.2)$ & \\
\hline Child-Pugh & & & 0.567 \\
\hline A & $54(93.1)$ & $19(100.0)$ & \\
\hline $\mathrm{B}$ & $4(6.9)$ & $0(0.0)$ & \\
\hline \multicolumn{4}{|c|}{ Continuous variables and ranked data } \\
\hline Age, years & & & 0.792 \\
\hline Median & 60.5 & 57 & \\
\hline Range & $30-77$ & $42-75$ & \\
\hline No. of tumors & & & 0.971 \\
\hline Median & 2 & 3 & \\
\hline Range & $1-6$ & $1-6$ & \\
\hline $\begin{array}{l}\text { Max diameter of } \\
\text { tumors, } \mathrm{mm}\end{array}$ & & & 0.184 \\
\hline Median & 52.5 & 44 & \\
\hline Range & $5-100$ & $15-100$ & \\
\hline ALT, U/L & & & 0.559 \\
\hline Median & 23.55 & 29.2 & \\
\hline Range & $8.2-238.8$ & $11.1-81.0$ & \\
\hline TBIL, $\mu \mathrm{mol} / \mathrm{L}$ & & & 0.110 \\
\hline Median & 14.6 & 12.3 & \\
\hline Range & $7.2-308.6$ & $1.8-25.5$ & \\
\hline
\end{tabular}

Table 1 (continued)

\begin{tabular}{|c|c|c|c|}
\hline $\begin{array}{l}\text { Demographics and } \\
\text { characteristics }\end{array}$ & $\begin{array}{c}\text { Primary } \\
\text { cohort }(n=58)\end{array}$ & $\begin{array}{c}\text { Validation } \\
\text { cohort }(n=19)\end{array}$ & $\begin{array}{l}\mathrm{P} \text { value of } \\
\text { difference }\end{array}$ \\
\hline Albumin, g/L & & & 0.307 \\
\hline Median & 43.3 & 40.9 & \\
\hline Range & 29.9-49.2 & $33-49.4$ & \\
\hline Prealbumin, g/L & & & 0.575 \\
\hline Median & 145.2 & 148.7 & \\
\hline Range & 25.8-276.9 & $56.3-242.3$ & \\
\hline ALP, U/L & & & 0.600 \\
\hline Median & 84.65 & 93.8 & \\
\hline Range & $13.1-416.8$ & $43.5-198.5$ & \\
\hline GGT, U/L & & & 0.132 \\
\hline Median & 48.3 & 65.55 & \\
\hline Range & $11.6-358.6$ & $10.4-318.2$ & \\
\hline MELD score & & & 0.822 \\
\hline Median & 8.3 & 7.9 & \\
\hline Range & $7.4-21.6$ & $6.1-14.2$ & \\
\hline $\mathrm{PT}, \mathrm{s}$ & & & 0.003 \\
\hline Median & 11.5 & 10.7 & \\
\hline Range & $9.8-14.1$ & $9.9-12.5$ & \\
\hline AFP, $\mu \mathrm{g} / \mathrm{L}$ & & & 0.829 \\
\hline Median & 6.245 & 5.05 & \\
\hline Range & $1.1-10,082$ & $1.16-1,461$ & \\
\hline CEA, $\mu \mathrm{g} / \mathrm{L}$ & & & 0.138 \\
\hline Median & 2.4 & 3.89 & \\
\hline Range & $0.58-415.5$ & $0.63-6.43$ & \\
\hline CA 19-9, U/mL & & & 0.417 \\
\hline Median & 18 & 48.1 & \\
\hline Range & $2.8-872.3$ & $8.87-573$ & \\
\hline \multicolumn{4}{|c|}{$\begin{array}{l}\text { ICC, intrahepatic cholangiocarcinoma; HCV, hepatitis C } \\
\text { virus; TACE, transcatheter arterial chemoembolization; } \\
\text { RFA, radiofrequency ablation; MWA, microwave ablation; } \\
\text { ALT, alanine aminotransferase; TBIL, total bilirubin; ALP, } \\
\text { alkaline phosphatase; GGT, gamma-glutamyl transferase; } \\
\text { PT, prothrombin time; AFP, alpha-fetoprotein; CEA, } \\
\text { carcinoembryonic antigen; CA 19-9, carbohydrate antigen 19-9. }\end{array}$} \\
\hline
\end{tabular}

Table 1 (continued) 
Table 2 Univariate and multivariate analysis of prognostic factors for primary cohort $(\mathrm{n}=58)$

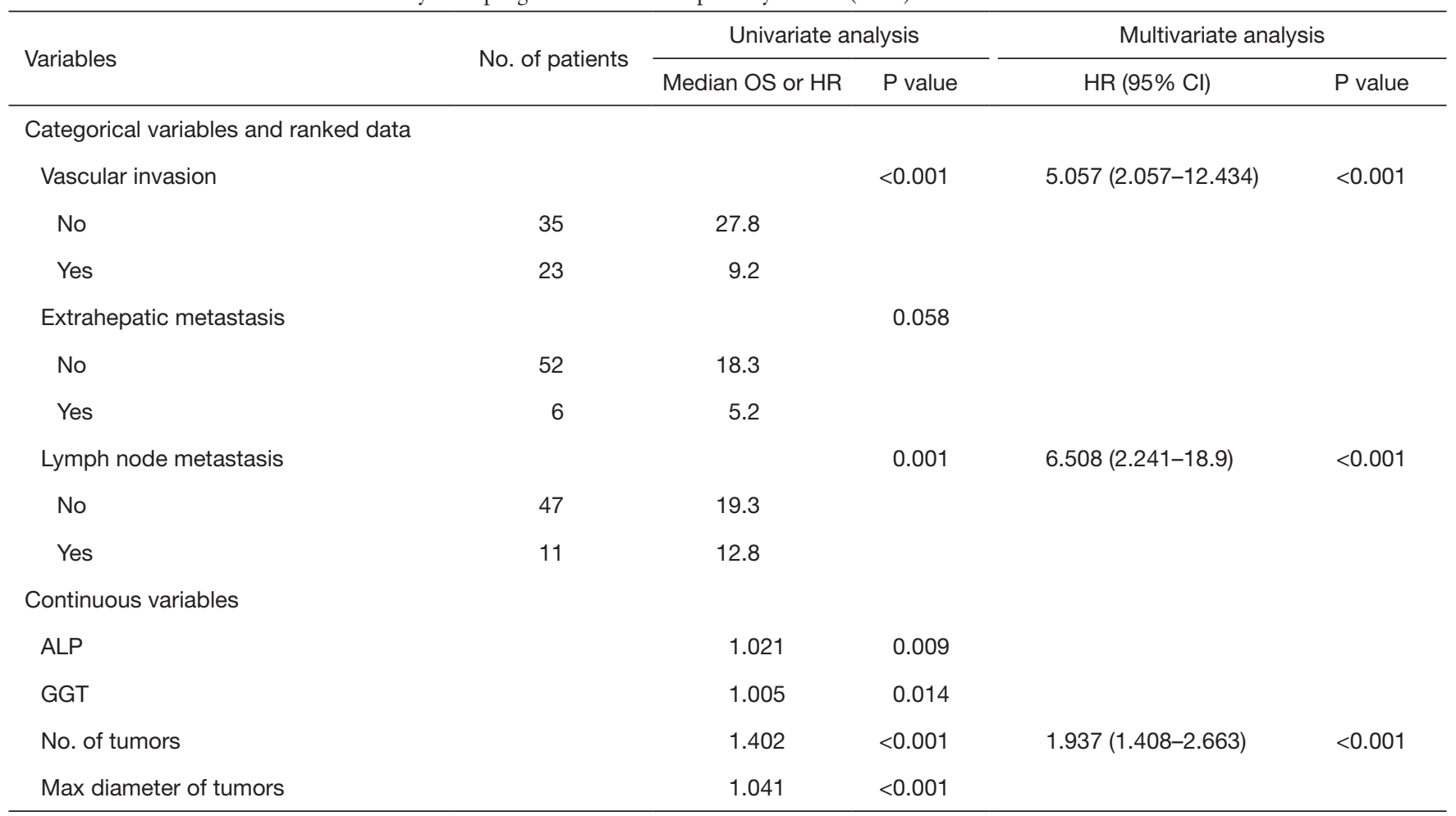

OS, overall survival; HR, hazard ratio; $\mathrm{Cl}$, confidence interval; ALP, alkaline phosphatase; GGT, gamma-glutamyl transferase.

\section{Univariate and multivariate analysis for OS of the primary cobort}

In the primary cohort, univariate analysis showed vascular invasion, lymph node metastasis, serum alkaline phosphatase (ALP) level, serum gamma-glutamyl transferase (GGT) level, number of tumors, and maximum diameter of tumors to be significantly associated with $\mathrm{OS}(\mathrm{P}<0.05$ for all; Table 2). Multivariate analysis revealed that only number of tumors, vascular invasion and lymph node metastasis were independent predictors of OS $(\mathrm{P}<0.001$ for all; Table 2).

\section{Prognostic nomogram for patients with ICC}

The nomogram to predict survival of ICC patients undergoing ablation was based on the results of multivariate analysis and integrated all three significant independent factors for OS (Figure 1). The predictive discrimination of the nomogram was evaluated using $\mathrm{C}$-index, which was 0.834 [95\% confidence interval (CI), 0.765-0.904]. The calibration plots showed favorable agreement between the prediction by the nomogram and the observed survival at 1 and 3 years after ablation (Figure $2 A, B$ ).

\section{Comparison of predictive power between the nomogram and number of tumors in the primary cobort}

As the only ordinal independent predictive factor, the number of tumors was used to construct a prognostic model. The C-index of this model for OS prediction was 0.731 (95\% CI, 0.643-0.820). Prediction of OS with this model was significantly less concordant with the observed outcome than that of the proposed nomogram $(\mathrm{P}<0.001)$.

\section{Comparison between the nomogram and currently available surgical prognostic systems}

Each current surgical prognostic system was applied to the primary cohort and the results were compared with that obtained with the nomogram. A standardized prognostic point was calculated according to the nomogram and each surgical staging/score system, with the highest stage/total score was counted as 100 points, and the lowest stage/ total score was counted as 0 point. Hazard ratios (HRs) for standardized prognostic point were calculated separately using the Cox regression method. Every prognostic system showed significant relevance with OS (Figure 3), with HRs 


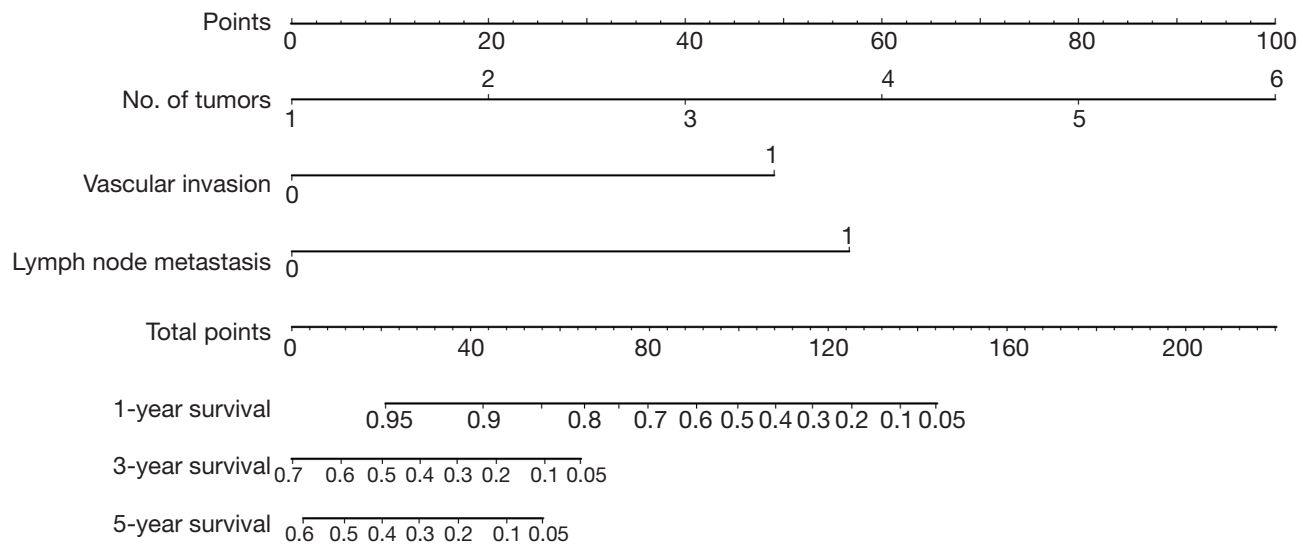

Figure 1 Prognostic nomogram for overall survival of patients with ICC undergoing ablation. To use the nomogram, each variable of an individual corresponds to a point on the points axis at the same vertical position. The total points were calculated and corresponds to the possibility of 1-, 3- and 5-year survival at the same vertical position. The 1-, 3- and 5-year survival probability could only be estimated, due to the non-linear distances of the values on the survival axes. Thus, the usage of survival probability range is suggested, for instance, 50-60\%, 70-75\%, 90-95\%. ICC, intrahepatic cholangiocarcinoma.
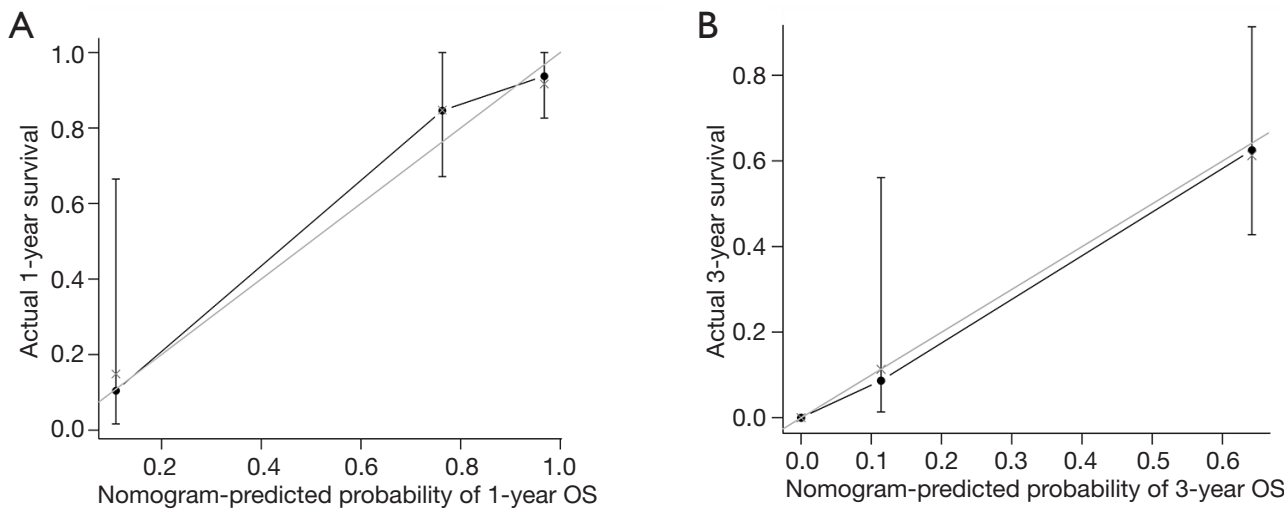

Figure 2 The calibration curve of nomogram predicted survival at (A) 1 year and (B) 3 years in the primary cohort. Nomogram-predicted survival probability is plotted on the $\mathrm{X}$-axis while actual OS is plotted on the Y-axis. Thin gray line represents the reference line. OS, overall survival.

all being $>1(\mathrm{P}<0.01$ for all). $\mathrm{C}$-indices for OS prediction were calculated for all surgical prognostic systems. The Wang nomogram showed an equivalent concordant prediction with the proposed nomogram $(\mathrm{P}>0.05)$, with C-index of 0.840 . All the other surgical prognostic systems were significantly less accurate in predicting survival than the proposed nomogram $(\mathrm{P}<0.01$ for all); these included the $7^{\text {th }}$ edition of the AJCC TNM classification (C-index, 0.763), the Nathan staging system (C-index, 0.686), the Okabayashi staging system (C-index, 0.691), the LCSGJ staging system (C-index, 0.702), the Uenishi staging system (C-index,
0.784), the Sakamoto staging system (C-index, 0.783), the Fudan score (C-index, 0.739), the Zhou score (C-index, 0.787), the Hyder nomogram (C-index, 0.795), and the Yeh nomogram (C-index, 0.749) (Figure 4).

\section{Predictive performance of the nomogram in the validation cohort}

In the validation cohort, the median OS was 14.5 months and the 1 -year OS rate was $69 \%$. The median PFS was 3.3 months, and the 1 -year PFS rate was $17 \%$. The $\mathrm{C}$-index 


Prognostic model
Proposed Nomogram
Fudan score
Zhou score
Wang nomogram
Hyder nomogram
Yeh nomogram
Okabayashi stage
LCSGJ stage
Nathan stage
7th AJCC stage
Uenishi stage
Sakamoto stag

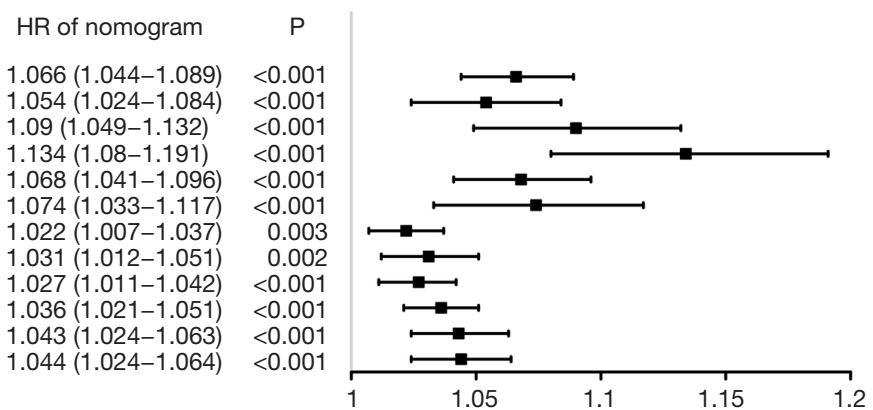

Figure 3 Forest plots to show the HRs of the total points calculated by different prognostic systems in the primary cohort. HR, hazard ratio.



Figure $4 \mathrm{C}$-indices of the proposed nomogram and the current prognostic systems for resection in the primary cohort.

of the nomogram was 0.839 (95\% CI, 0.731-0.947). In comparison, the $\mathrm{C}$-index of the prognostic model based on the number of tumors was 0.792 (95\% CI, 0.658-0.926). Concordance with the observed survival was significantly better with the nomogram $(\mathrm{P}<0.001)$.

\section{Discussion}

Percutaneous thermal ablation and TACE have been widely applied to treat liver cancer. At our institution, we performed pre-operative TACE by using lipiodol as embolization agent, and lipiodol deposition in the tumorfeeding artery could be perfect target for tumor location under CT guidance. For large and multifocal tumors, ablation therapy is usually performed in sequential multiple sessions to induce larger tumor necrosis volume. Limited data are available for ICC patients treated by thermal ablation and TACE. In a previous study by Haidu et al., ablation treatment induced a 3 -year OS rate of $71 \%$, which was quite promising for ICC patients. This could be owed to the high technical effectiveness rate (92\%) (25). In their study, the ablation treatment was aggressive, and patients with large size and number of tumors were included. The survival outcomes were favorable, but the major complication rate was high (13\%). And the sample size was small (11 patients), which might limit the force of the 
statistics. In our study, median OS for the primary cohort and validation cohort were 17.9 and 14.5 months $(\mathrm{P}>0.05)$, respectively. For patients with resectable ICC in the primary cohort, thermal ablation showed a curative efficacy (median OS, 27.8 months; 5-year OS rate, 38.5\%). For patients with resectable tumor in the entire cohort, median OS was 27.8 months and 5 -year OS rate was $39.3 \%$. The results indicated that thermal ablation could provide a favorable long-term survival in ICC patients. In previous reports, median survival of ICC patients treated with surgical resection was reported to be $14.8-45$ months, and 5-year OS rate $18-43.2 \%$ (26). The efficacy of thermal ablation appears promising, yet remains to be further evaluated in different populations of ICC patients. On the other hand, median OS of unresectable ICC was 12.7 months, and the 1 -year OS rate was $50.6 \%$, suggesting that ablation is rather an effective approach for systemic disease burden than a 'cure' for unresectable individuals.

Patients with unresectable ICC had median OS of 12.7 months. The tumors in these patients were ineligible for resection and refractory to other therapies (including chemotherapy and radiation). In our department we have been successful in curing large and multiple tumors using TACE plus ablation, thus these patients were also treated. Although patients with unresectable ICC were reported to have extremely poor prognosis if untreated, our results indicated that thermal ablation and TACE might be a promising therapy for patients unsuitable for surgery. Though not as wide spread as surgical treatment, local ablation + TACE showed promising therapeutic results and draw attention for further research.

TACE is usually used as adjuvant therapy combined with ablation (27) or as a palliative modality. The chemotherapeutic agents and embolization of the tumor feeding artery could have positive effects upon survival. In the current study, TACE showed no significant relevance with survival, and there might be at least two reasons as follows: (I) tumor necrosis was mainly induced by thermal ablation in this study; thus the relation of TACE with survival might not be as significant as that of thermal ablation; (II) only 10 patients underwent thermal ablation without pre-ablation TACE, and the limited number of patients might not be enough to yield significant difference.

Current prognostic systems of ICC are controversial and less than optimal, and prognosis of ICC patient is difficult to be accurately evaluated. For patients with ICC, especially for those undergoing thermal ablation treatment, the primary usage of the nomogram is to calculate the 1-,
3-, 5-year survival probability after treatment, which might be the answer to the major concern of both patients and clinicians. Eleven prognostic systems, including the TNM staging systems, score systems, and prognostic nomograms, are currently available. The $7^{\text {th }}$ edition of the AJCC Staging Manual introduced a staging system approximately the same as that of HCC, and the staging system is still under modifying in updated versions of the AJCC manual. An analysis by Kim et al. showed that the C-index for OS was 0.669 for the $8^{\text {th }}$ edition and 0.667 for the $7^{\text {th }}$ edition AJCC staging systems, respectively (28). However, C-index lower than 0.7 does not indicate a favorable predictive ability.

Moreover, some aspects of these prognostic systems are controversial (29); for example, doubts have been raised about (I) whether tumor diameter should be included in the prognostic system; (II) whether numerical data such as tumor number has a linear association with the likelihood of mortality; (III) whether additional factors such as CA199 and age should be taken into consideration; (IV) whether complex prognostic nomograms are feasible to use; and (V) whether these prognostic systems, which are designed for patients undergoing hepatectomy could be applied for patient treated by other therapies such as thermal ablation. In the present study tumor diameter was not an independent predictor of survival in multivariate analysis. This did not necessarily indicate that tumor diameter had none effect on survival, since it did show a significant effect as a linear variable on univariate analysis $(\mathrm{P}<0.001)$. Furthermore, during the bidirectional elimination process with the Akaike information criterion, tumor diameter was the last variable excluded from the final model, indicating that tumor diameter might have potential effect on survival, although maybe not as significant as that of the three factors in the multivariate model. In several prognostic nomograms of other types of cancer, tumor size/diameter was found significantly related with survival probability (30). Taken together, tumor size is still an important potential factor to predict post-treatment survival.

None of the currently available prognostic systems used the number of tumors as an ordinal prognostic variable. The traditional TNM staging systems used it as a categorical variable, i.e., solitary tumor or multiple tumors (more than one tumor), as also did the Fudan score, the Zhou score, and the Hyder nomogram. These prognostic systems did not further explore the potential prognostic value of different tumor numbers. The Wang nomogram divided the number of tumors into three categories: 1 , $2-3$, and $>3$. However, the rationale and statistical analysis 
process of the cut-off points were unavailable in their study. We hypothesized that greater number of tumors would indicate worse prognosis. In our study, for patients with 1, 2, $3,4,5,6$ or more tumors, the median OS were $25.5(n=25)$, $27.8(\mathrm{n}=14), 12.8(\mathrm{n}=14), 5.2(\mathrm{n}=3), 4.5(\mathrm{n}=7), 9.2(\mathrm{n}=14)$ months, respectively. The results showed that OS tended to decrease as tumor number increased. However, the survival of patients with 4 and 5 tumors were less than those with 6 tumors, and this might be due to the small number of patients with $4(n=3)$ and $5(n=7)$ tumors. Further subgroup analysis showed that the $75 \%$ survival time of patients $(n=37)$ without vascular invasion or lymph node metastasis were 20.9, 27.8, 18.3, NA, 16.5 and 0 , for patients with 1, 2, 3, 4, 5,6 or more tumors, respectively, indicating an approximate linear relationship between the tumor number and survival, in general. Further research is needed to clarify the effect of tumor number on OS.

This nomogram included lymph nodes metastasis, which was diagnosed by imaging method in this study. The diagnostic accuracy of a single CT imaging might be unfavorable, with high misdiagnosis rates. However, one of the diagnostic tools used in this study, FDG-PET/PET-CT, has been reported to show excellent diagnostic performance for detecting lymph node metastases in most types of tumors, including cholangiocarcinoma in many researches. On the other hand, due to the innate characteristics of this retrospective study, sequential pre-operative and postoperative CT imagings of ICC patients are available, and lymph nodes metastases could be retrospectively diagnosed by these sequential CT imagings, based on typical manifestations such as increasing size and numbers within weeks or months. Thus, for patients infeasible for biopsy, diagnosis of lymph nodes metastasis by the methods in this study might be also effective and helpful to evaluate prognosis. The inclusion of lymph nodes metastasis in this nomogram might be necessary and helpful.

Patients with tumor recurrence were also included. Though inclusion of recurrent ICC patients might lead to more heterogeneous sample, these patients were still included in this study. Substantial numbers of recurrent ICC patients are unsuitable for re-resection, thus might seek help from interventional therapists. As a safe and effective alternative, local regional ablation (with TACE) therapies are also commonly performed on recurrent ICC patients. Inclusion of patients with post-resection recurrence makes this nomogram also applicable for recurrent ICC patients.

The ideal nomogram needs to be both simple and accurate. Unlike the three currently available nomograms, the proposed nomogram is based on only three imaging characteristics and should therefore be easy to apply in the average patient. The prognostic accuracy was also good. The calibration plot (Figure 2A,B) demonstrated the agreement between predictive outcome and actual observation. However, it should be noted that the nomogram showed more stable predictive performance at a shorter time of follow-up. As shown in Figure 2, the standard error bars at 1-year (Figure 2A) were shorter than those at 3-year (Figure 2B). The nomogram and the $\mathrm{C}$-index would be more convincing when assessing shorttime survival. All current prognostic systems were evaluated using the primary cohort (Figure 4). Except for the Wang nomogram, all the other models were significantly less accurate than the proposed nomogram $(\mathrm{P}<0.01)$, and their $\mathrm{C}$-indices were much lower (ranging from 0.686 to 0.794 ). Notably, the C-index of Wang nomogram (0.840; 95\% CI, $0.744-0.936)$ was nearly the same as that of the proposed nomogram. The reason may be that both nomogram were based on data from Chinese populations, and the prognostic model form of nomogram could present more specific prognostic information than simplified staging systems. However, the Wang nomogram included serum tumor markers [carcinoembryonic antigen (CEA) and carbohydrate antigen 19-9 (CA19-9)] as linear prognostic factors. Only a few studies have reported significant association of these factors with prognosis (31). The controversies of cut-off values of continuous variables need to be further clarified and the complexity of the calculation may be a limitation.

This study has several limitations. Due to the low incidence of ICC, the number of patients in this study was relatively small. Secondly, number of patients with extrahepatic metastasis was small and part of these patients lost follow-up, thus extrahepatic metastasis failed to show a statistically significant influence on survival. This important potential prognostic factor should be further investigated in the following research works. Third, due to the retrospective nature of the study, there might be bias in patient selection. For instance, the rates of vascular invasion and metastasis are high, since patients with more advanced disease are usually unsuitable for surgical resection, thus might seek help from less invasive treatments such as thermal ablation. As a result, the therapeutic value of thermal ablation might be underestimated. Further prospective comparison of thermal ablation and other therapies with more rigorous inclusion criteria, especially the disease stage of ICC, are needed. 


\section{Conclusions}

In conclusion, percutaneous thermal ablation appears to be effective for ICC patients. The proposed nomogram is suitable for ICC patients undergoing ablation. Further external studies are needed to validate this nomogram on large samples.

\section{Acknowledgments}

Funding: This study was supported by Capital's Funds for Health Improvement and Research (CFH 2020-2-2175); Beijing Talents Project, Funding for High-level Talents in Beijing Municipal Health System (2014-3-088); National Major Scientific Instruments and Equipment Development Project (ZDYZ2015-2); and Beijing You'an Hospital Hepatic Disease \& HIV Fund (20150203).

\section{Footnote}

Reporting Checklist: The authors have completed the TRIPOD reporting checklist. Available at http://dx.doi. org/10.21037/tcr-20-2299

Data Sharing Statement: Available at http://dx.doi. org/10.21037/tcr-20-2299

Conflicts of Interest: All authors have completed the ICMJE uniform disclosure form (available at http://dx.doi. org/10.21037/tcr-20-2299). The authors have no conflicts of interest to declare.

Ethical Statement: The authors are accountable for all aspects of the work in ensuring that questions related to the accuracy or integrity of any part of the work are appropriately investigated and resolved. The study was conducted in accordance with the Declaration of Helsinki (as revised in 2013) and the Harmonized Tripartite Guideline for Good Clinical Practice from the International Conference on Harmonization. This study was reviewed and approved by the Beijing You'an Hospital Ethics Committee [approval number 2017(28)]. All patients enrolled completed the informed consent form.

Open Access Statement: This is an Open Access article distributed in accordance with the Creative Commons Attribution-NonCommercial-NoDerivs 4.0 International License (CC BY-NC-ND 4.0), which permits the non- commercial replication and distribution of the article with the strict proviso that no changes or edits are made and the original work is properly cited (including links to both the formal publication through the relevant DOI and the license). See: https://creativecommons.org/licenses/by-nc-nd/4.0/.

\section{References}

1. Razumilava N, Gores GJ. Cholangiocarcinoma. Lancet 2014;383:2168-79.

2. Rizvi S, Gores GJ. Pathogenesis, diagnosis, and management of cholangiocarcinoma. Gastroenterology 2013;145:1215-29.

3. Bergquist A, von Seth E. Epidemiology of cholangiocarcinoma. Best Pract Res Clin Gastroenterol 2015;29:221-32.

4. Khan SA, Emadossadaty S, Ladep NG, et al. Rising trends in cholangiocarcinoma: is the ICD classification system misleading us? J Hepatol 2012;56:848-54.

5. Khan SA, Davidson BR, Goldin RD, et al. Guidelines for the diagnosis and treatment of cholangiocarcinoma: an update. Gut 2012;61:1657-69.

6. Wang Y, Li J, Xia Y, et al. Prognostic nomogram for intrahepatic cholangiocarcinoma after partial hepatectomy. J Clin Oncol 2013;31:1188-95.

7. Okabayashi T, Yamamoto J, Kosuge T, et al. A new staging system for mass-forming intrahepatic cholangiocarcinoma: analysis of preoperative and postoperative variables. Cancer 2001;92:2374-83.

8. Vauthey JN, Lauwers GY, Esnaola NF, et al. Simplified staging for hepatocellular carcinoma. J Clin Oncol 2002;20:1527-36.

9. Nathan H, Aloia TA, Vauthey JN, et al. A proposed staging system for intrahepatic cholangiocarcinoma. Ann Surg Oncol 2009;16:14-22.

10. Jiang $W$, Zeng ZC, Tang ZY, et al. A prognostic scoring system based on clinical features of intrahepatic cholangiocarcinoma: the Fudan score. Ann Oncol 2011;22:1644-52.

11. Uenishi T, Ariizumi S, Aoki T, et al. Proposal of a new staging system for mass-forming intrahepatic cholangiocarcinoma: a multicenter analysis by the Study Group for Hepatic Surgery of the Japanese Society of Hepato-Biliary-Pancreatic Surgery. J Hepatobiliary Pancreat Sci 2014;21:499-508.

12. Hyder O, Marques H, Pulitano C, et al. A nomogram to predict long-term survival after resection for intrahepatic cholangiocarcinoma: an Eastern and Western experience. 
JAMA Surg 2014;149:432-8.

13. Yeh CN, Wang SY, Chen YY, et al. a prognostic nomogram for overall survival of patients after hepatectomy for intrahepatic cholangiocarcinoma. Anticancer Res 2016;36:4249-58.

14. Park J, Kim MH, Kim KP, et al. Natural history and prognostic factors of advanced cholangiocarcinoma without surgery, chemotherapy, or radiotherapy: a largescale observational study. Gut Liver 2009;3:298-305.

15. Wang Y, Ma L, Sheng S, et al. Combination therapy of TACE and CT-guided partial hepatic segment ablation for liver cancer. Minim Invasive Ther Allied Technol 2018;27:355-64.

16. Rizzo A, Ricci AD, Tober N, et al. Second-line treatment in advanced biliary tract cancer: today and tomorrow. Anticancer Res 2020;40:3013-30.

17. Han K, Ko HK, Kim KW, et al. Radiofrequency ablation in the treatment of unresectable intrahepatic cholangiocarcinoma: systematic review and meta-analysis. J Vasc Interv Radiol 2015;26:943-8.

18. Brandi G, Rizzo A, Dall'Olio FG, et al. Percutaneous radiofrequency ablation in intrahepatic cholangiocarcinoma: a retrospective single-center experience. Int J Hyperthermia 2020;37:479-85.

19. Edge SB, Compton CC. The American Joint Committee on Cancer: the 7th edition of the AJCC cancer staging manual and the future of TNM. Ann Surg Oncol 2010;17:1471-4.

20. Yamasaki S. Intrahepatic cholangiocarcinoma: macroscopic type and stage classification. J Hepatobiliary Pancreat Surg 2003;10:288-91.

21. Sakamoto Y, Kokudo N, Matsuyama Y, et al. Proposal of a new staging system for intrahepatic cholangiocarcinoma: analysis of surgical patients from a nationwide survey of the Liver Cancer Study Group of Japan. Cancer 2016;122:61-70.

22. Zhou H, Jiang X, Li Q, et al. A simple and effective

Cite this article as: Wang Y, Yuan Z, Liu B, Chen H, Yin T, Zheng J, Li W. Long-term outcome and prognostic nomogram for intrahepatic cholangiocarcinoma after thermal ablation: a retrospective study. Transl Cancer Res 2020;9(11):6743-6754. doi: 10.21037/tcr-20-2299 prognostic staging system based on clinicopathologic features of intrahepatic cholangiocarcinoma. Am J Cancer Res 2015;5:1831-43.

23. Schulick RD. Criteria of unresectability and the decisionmaking process. HPB (Oxford) 2008;10:122-5.

24. Harrell FE Jr, Lee KL, Mark DB. Multivariable prognostic models: issues in developing models, evaluating assumptions and adequacy, and measuring and reducing errors. Stat Med 1996;15:361-87.

25. Haidu M, Dobrozemsky G, Schullian P, et al. Stereotactic radiofrequency ablation of unresectable intrahepatic cholangiocarcinomas: a retrospective study. Cardiovasc Intervent Radiol 2012;35:1074-82.

26. Choi SB, Kim KS, Choi JY, et al. The prognosis and survival outcome of intrahepatic cholangiocarcinoma following surgical resection: association of lymph node metastasis and lymph node dissection with survival. Ann Surg Oncol 2009;16:3048-56.

27. Liu B, Jiang C, Chen S, et al. Multipronged ethanol ablation combined with TACE for intermediate hepatocellular carcinoma. Minim Invasive Ther Allied Technol 2018;27:300-8.

28. Kim Y, Moris DP, Zhang XF, et al. Evaluation of the 8th edition American Joint Commission on Cancer (AJCC) staging system for patients with intrahepatic cholangiocarcinoma: A surveillance, epidemiology, and end results (SEER) analysis. J Surg Oncol 2017;116:643-50.

29. Hatzaras I, Schmidt C, Muscarella P, et al. Elevated CA 19-9 portends poor prognosis in patients undergoing resection of biliary malignancies. HPB (Oxford) 2010;12:134-8.

30. Wang Y, Zheng J, Chen H, et al. A prognostic nomogram for colorectal cancer liver metastases after percutaneous thermal ablation. Int J Hyperthermia 2018;34:853-62.

31. Cho SY, Park SJ, Kim SH, et al. Survival analysis of intrahepatic cholangiocarcinoma after resection. Ann Surg Oncol 2010;17:1823-30. 